\title{
Cusp effects in meson decays
}

\author{
Bastian Kubis, ${ }^{\text {a }}$ \\ Helmholtz-Institut für Strahlen- und Kernphysik (Theorie) and Bethe Center for Theoretical Physics, Universität Bonn, \\ D-53115 Bonn, Germany
}

\begin{abstract}
The pion mass difference generates a pronounced cusp in the $\pi^{0} \pi^{0}$ invariant mass distribution of $K^{+} \rightarrow \pi^{0} \pi^{0} \pi^{+}$decays. As originally pointed out by Cabibbo, an accurate measurement of the cusp may allow one to pin down the S-wave pion-pion scattering lengths to high precision. We present the non-relativistic effective field theory framework that permits to determine the structure of this cusp in a straightforward manner, including the effects of radiative corrections. Applications of the same formalism to other decay channels, in particular $\eta$ and $\eta^{\prime}$ decays, are also discussed.
\end{abstract}

\section{The pion mass and pion-pion scattering}

The approximate chiral symmetry of the strong interactions severely constrains the properties and interactions of the lightest hadronic degrees of freedom, the would-be Goldstone bosons (in the chiral limit of vanishing quark masses) of spontaneous chiral symmetry breaking that can be identified with the pions. The effective field theory that systematically exploits all the consequences that can be derived from symmetries is chiral perturbation theory $[1,2]$, which provides an expansion of low-energy observables in terms of small quark masses and small momenta.

One of the most elementary consequences of chiral symmetry is the well-known Gell-Mann-Oakes-Renner relation [3] for the pion mass $M$ in terms of the light quark masses (at leading order),

$$
M^{2}=B\left(m_{u}+m_{d}\right), \quad B=-\frac{\langle 0|\bar{u} u| 0\rangle}{F^{2}} .
$$

A non-vanishing order parameter $B$, related to the light quark condensate via the pion decay constant $F$ (in the chiral limit), is a sufficient (but not necessary) condition for chiral symmetry breaking. Chiral perturbation theory allows to calculate corrections to this relation [2],

$$
M_{\pi}^{2}=M^{2}-\frac{M^{4}}{32 \pi^{2} F^{2}} \bar{\ell}_{3}+O\left(M^{6}\right),
$$

with the a priori unknown low-energy constant $\bar{\ell}_{3}$. Another way to write Eq. (2) is therefore

$$
M_{\pi}^{2}=B\left(m_{u}+m_{d}\right)+A\left(m_{u}+m_{d}\right)^{2}+O\left(m_{u, d}^{3}\right),
$$

and the natural question arises: how do we know that the leading term in the quark-mass expansion of $M_{\pi}^{2}$ really dominates the series? $\bar{\ell}_{3}$ could actually be anomalously large, the consequence of which has been explored as an

\footnotetext{
a e-mail: kubis@hiskp.uni-bonn.de
}

alternative scenario of chiral symmetry breaking under the name of generalized chiral perturbation theory [4].

Fortunately, chiral low-energy constants tend to appear in more than one observable, and indeed, $\bar{\ell}_{3}$ also features in the next-to-leading-order corrections to the isospin $I=0$ S-wave pion-pion scattering length $a_{0}^{0}$ [2],

$$
\begin{aligned}
a_{0}^{0} & =\frac{7 M_{\pi}^{2}}{32 \pi F_{\pi}^{2}}\left\{1+\epsilon+O\left(M_{\pi}^{4}\right)\right\}, \\
\epsilon & =\frac{5 M_{\pi}^{2}}{84 \pi^{2} F_{\pi}^{2}}\left(\bar{\ell}_{1}+2 \bar{\ell}_{2}-\frac{3}{8} \bar{\ell}_{3}+\frac{21}{10} \bar{\ell}_{4}+\frac{21}{8}\right) .
\end{aligned}
$$

$\bar{\ell}_{1}$ and $\bar{\ell}_{2}$ are known from $\pi \pi \mathrm{D}$-waves $a_{2}^{0}, a_{2}^{2}$, while $\bar{\ell}_{4}$ can be determined from a dispersive analysis of the scalar radius of the pion $\left\langle r^{2}\right\rangle_{\pi}^{S}[5,6]$, such that the correction term $\epsilon$ in Eq. (4) can be rewritten as

$$
\epsilon=M_{\pi}^{2}\left\{\frac{\left\langle r^{2}\right\rangle_{\pi}^{S}}{3}+\frac{200 \pi}{7} F_{\pi}^{2}\left(a_{2}^{0}+2 a_{2}^{2}\right)-\frac{15 \bar{\ell}_{3}-353}{672 \pi^{2} F_{\pi}^{2}}\right\} .
$$

Consequently, a measurement of $a_{0}^{0}$ can lead to a determination of $\bar{\ell}_{3}$, and hence to a clarification of the role of the various order parameters of chiral symmetry breaking in nature. We wish to point out that Eq. (5) only rewrites the dependence of $a_{0}^{0}$ on the $O\left(p^{4}\right)$ low-energy constants $\bar{\ell}_{1-4}$ in the form of a low-energy theorem. The theoretical predictions of the two S-wave $\pi \pi$ scattering lengths of isospin 0 and 2 from a combination of two-loop chiral perturbation theory [7,6] and a Roy equation analysis [8] (for QCD in the isospin limit),

$$
\begin{aligned}
a_{0}^{0} & =0.220 \pm 0.005, \\
a_{0}^{2} & =-0.0444 \pm 0.0010, \\
a_{0}^{0}-a_{0}^{2} & =0.265 \pm 0.004,
\end{aligned}
$$

do not depend on the D-wave scattering lengths as input, but rather yield values for all $\pi \pi$ threshold parameters as results. The predictions Eq. (6) are among the most precise in 
low-energy hadron physics and present a formidable challenge for experimental verification. For other recent phenomenological determinations of the scattering lengths, see Refs. [9-11].

Traditionally, information on pion-pion scattering has been extracted from reactions on nucleons, which is difficult to achieve in a model-independent way, and data are usually not available very close to threshold kinematics. The latest precision determinations therefore mainly concern three different methods: the lifetime measurement of pionium [12], $K_{e 4}$ decays $[13,14]$, and, most recently, the so-called cusp effect in $K \rightarrow 3 \pi$ decays.

Let us very briefly discuss the first two modern experimental approaches. Pionium is the electromagnetically bound state of a $\pi^{+} \pi^{-}$pair, with an ionization energy of about $1.86 \mathrm{keV}$ and a ground state width of about $0.2 \mathrm{eV}$. Its energy levels as given by purely electromagnetic binding are perturbed by the short-ranged strong interactions: they are shifted by elastic strong rescattering $\pi^{+} \pi^{-}$, but in particular, even the ground state is not stable, it decays dominantly into $\pi^{0} \pi^{0}$. The decay width is given by the following (improved) Deser formula $[15,16]$

$$
\Gamma=\frac{2}{9} \alpha^{3} p\left|a_{0}^{0}-a_{0}^{2}\right|^{2}(1+\delta),
$$

where $\alpha$ is the fine structure constant, $p$ the momentum of a final-state $\pi^{0}$ in the center-of-mass frame, and $\delta$ is a numerical correction factor accounting for isospin violation beyond leading order, $\delta=0.058 \pm 0.012$ [17]. Given the theoretical values for the $\pi \pi$ scattering lengths of Eq. (6), the pionium lifetime can be predicted to be

$$
\tau=(2.9 \pm 0.1) \times 10^{-15} s,
$$

while ultimately, the argument should be reversed, and a measurement of the lifetime is to be used for a determination of $a_{0}^{0}-a_{0}^{2}$. The current value from the DIRAC experiment [12],

$$
\tau=\left(2.91_{-0.62}^{+0.49}\right) \times 10^{-15} s,
$$

agrees with Eq. (8), but is not yet comparably precise. For a comprehensive review of the theory of hadronic atoms, see Ref. [18].

The decay $K^{+} \rightarrow \pi^{+} \pi^{-} e^{+} v_{e}\left(K_{e 4}\right)$ can be described in terms of hadronic form factors, which, in the isospin limit, share the phases of $\pi \pi$ scattering due to Watson's final state theorem [19]. What can be extracted unambiguously from the decay, using the so-called Pais-Treiman method [20], is the difference of $\pi \pi I=0 \mathrm{~S}$-wave and $I=1 \mathrm{P}$-wave phase shifts

$$
\delta_{0}^{0}\left(s_{\pi \pi}\right)-\delta_{1}^{1}\left(s_{\pi \pi}\right),
$$

and as the energy of the two pions is kinematically restricted to $\sqrt{s_{\pi \pi}}<M_{K}$, these phases are accessible close to threshold. It has been pointed out [21] that, given the precision of the latest NA48/2 data [14], it is necessary to include an isospin-breaking correction phase in the analysis. The resulting scattering length determination will be shown in the comparison in Sect. 4.

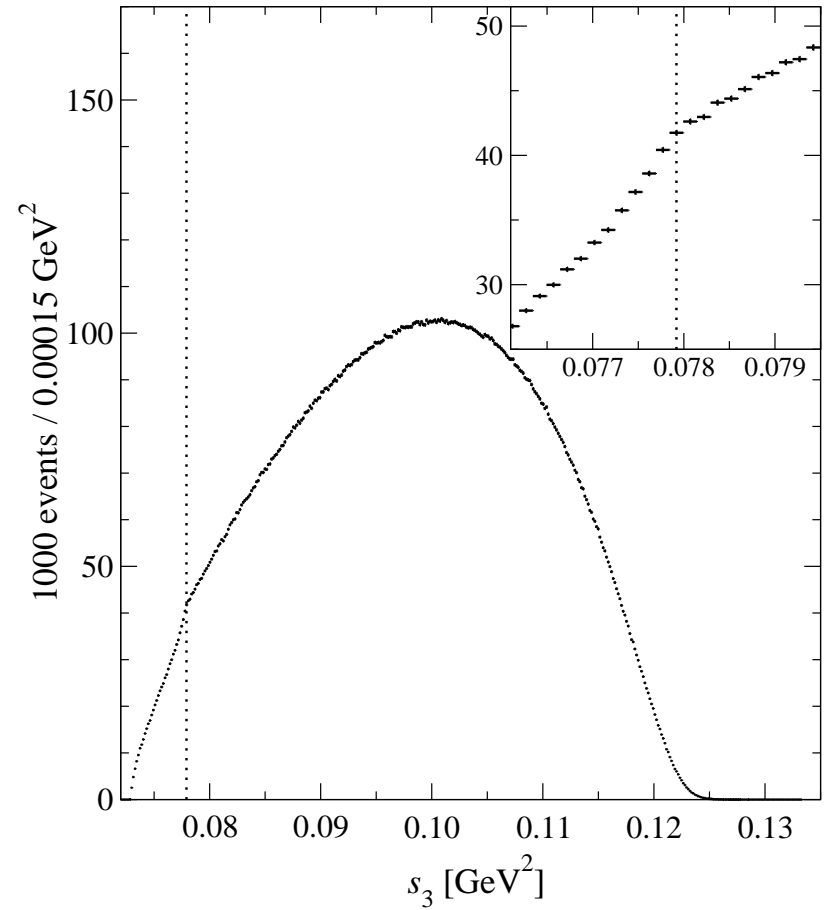

Fig. 1. Cusp in the decay spectrum $d \Gamma / d s_{3}$ of the decay $K^{ \pm} \rightarrow$ $\pi^{0} \pi^{0} \pi^{ \pm}$as seen by the NA48/2 collaboration. The dotted vertical line marks the position of the $\pi^{+} \pi^{-}$threshold, the insert focuses on the cusp region. Data taken from Ref. [22].

\section{The cusp effect in $K^{ \pm} \rightarrow \pi^{0} \pi^{0} \pi^{ \pm}$decays}

In an investigation of the decay $K^{ \pm} \rightarrow \pi^{0} \pi^{0} \pi^{ \pm}$, the NA48/2 collaboration at CERN has observed a cusp, i.e. a sudden, discontinuous change in slope, in the decay spectrum with respect to the invariant mass squared of the $\pi^{0} \pi^{0}$ pair $d \Gamma / d s_{3}, s_{3}=M_{\pi^{0} \pi^{0}}^{2}[22]$; see Fig. 1. A first qualitative explanation was subsequently given by Cabibbo [23], who pointed out that a $K^{+}$can, simplistically speaking, either decay "directly" into the $\pi^{0} \pi^{0} \pi^{+}$final state, or alternatively decay into three charged pions $\pi^{+} \pi^{+} \pi^{-}$, with a $\pi^{+} \pi^{-}$pair rescattering via the charge-exchange process into two neutral pions, compare Fig. 2. The loop (rescattering) diagram has a non-analytic piece proportional to

$$
i v_{ \pm}\left(s_{3}\right)= \begin{cases}i \sqrt{1-\frac{4 M_{\pi^{+}}^{2}}{s_{3}}}, & s_{3}>4 M_{\pi^{+}}^{2}, \\ -\sqrt{\frac{4 M_{\pi^{+}}^{2}}{s_{3}}-1}, & s_{3}<4 M_{\pi^{+}}^{2},\end{cases}
$$

and as the charged pion is heavier than the neutral one by nearly $4.6 \mathrm{MeV}$, the (then real) loop diagram can interfere with the "direct" decay below the $\pi^{+} \pi^{-}$threshold and produce a square-root-like singularity at $s_{3}=4 M_{\pi^{+}}^{2}$, the cusp visible in the experimentally measured spectrum Fig. 1. Such threshold singularities have of course been known for a long time [24] and have been re-discovered for the scattering of neutral pions in the context of chiral perturbation theory [25]. It had even been pointed out very early by Budini and Fonda [26] that these cusps may be used to investigate $\pi \pi$ scattering: as suggested in Fig. 2, the strength of 

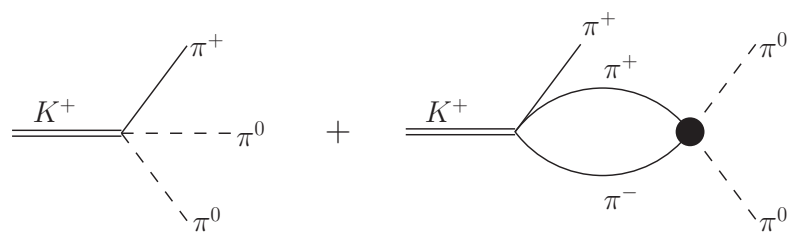

Fig. 2. "Direct" and "rescattering" contribution to the decay $K^{+} \rightarrow \pi^{0} \pi^{0} \pi^{+}$. The black dot marks the charge-exchange $\pi \pi$ scattering vertex proportional to the scattering lengths at threshold.

the cusp is proportional to the charge-exchange pion-pion scattering amplitude at threshold, hence a combination of scattering lengths might be extracted from a precision analysis of the cusp effect.

The challenge for theory is to provide a framework that matches the tremendous accuracy of the experimental data: the partial data sample analyzed in Ref. [22] was based on $2.3 \times 10^{7} K^{ \pm} \rightarrow \pi^{0} \pi^{0} \pi^{ \pm}$decays, subsequently expanded to more than $6.0 \times 10^{7}$ decays [27]. Different theoretical approaches have been suggested to this end: a combination of analyticity and unitarity with an expansion of the rescattering effects in powers of the $\pi \pi$ threshold parameters [28], and chiral perturbation theory beyond one-loop order [29]. In the following, we advocate the use of non-relativistic effective field theory [30] as the appropriate systematic tool to analyze these decays.

\section{Non-relativistic effective field theory}

Consider as a starting point a generic $\pi \pi$ partial wave amplitude $T$. Close to threshold, its real part can be written in the effective range expansion according to

$$
\operatorname{Re} T=a+b q^{2}+c q^{4}+\ldots
$$

with the scattering length $a$, the effective range $b$, a shape parameter $c$ etc. Chiral perturbation theory allows to calculate the parameters $a, b, c$ to a certain accuracy in the quark-mass (or pion-mass) expansion, see Eq. (4) for an example, but in principle, each of these parameters receives contributions from each loop order. On the other hand, one can set up a non-relativistic effective field theory (NREFT) in such a way that the scattering length $a$ is entirely given in terms of tree graphs, without any further loop corrections; similarly, the effective range $b$ can be calculated from tree and two-loop graphs only, but then no further contributions. In other words, NREFT allows to parameterize $T$ directly in terms of threshold parameters. Note that this is exactly what we want: the aim here is not to predict the scattering lengths, but to provide a representation of the (scattering or decay) amplitude in terms of the $\pi \pi$ threshold parameters that allows for an accurate extraction of the latter from experimental data. This is similar in spirit to the use of NREFT in the analysis of hadronic atoms in order to extract scattering lengths of different systems from their life times or energy level shifts (see Ref. [18] and references therein).

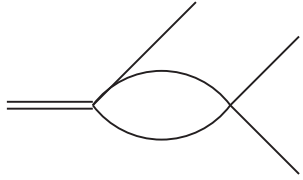

(a)
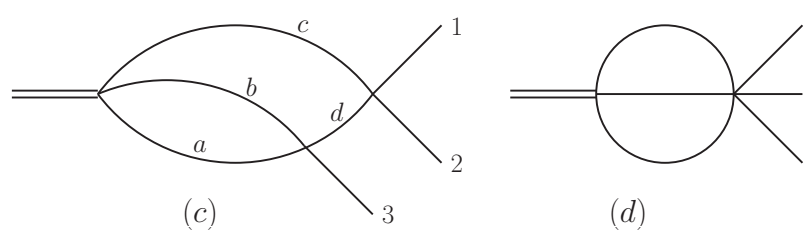

$(d)$
Fig. 3. Topologies for $\pi \pi$ rescattering graphs at one and two loops. The double line denotes the decaying kaon, while single lines stand for either charged or neutral pions.

\subsection{Power counting, Lagrangians}

First, we need to specify our power counting scheme. We introduce a formal non-relativistic parameter $\epsilon$ and count 3-momenta of the pions in the final state according to $|\mathbf{p}| / M_{\pi}=O(\epsilon)$. Consequently, the pions' kinetic energies are

$$
T_{i}=\omega_{i}\left(\mathbf{p}_{i}\right)-M_{i}=O\left(\epsilon^{2}\right), \text { where } \omega_{i}\left(\mathbf{p}_{i}\right)=\sqrt{M_{i}^{2}+\mathbf{p}_{i}^{2}},
$$

with $i=1,2,3, M_{1}=M_{2}=M_{\pi^{0}}, M_{3}=M_{\pi^{+}}$, and the $Q$-value of the reaction has to be counted as $O\left(\epsilon^{2}\right)$, too, as

$$
M_{K}-\sum_{i} M_{i}=\sum_{i} T_{i}=O\left(\epsilon^{2}\right) .
$$

In addition, we adopt the suggestion of Ref. [28]: as the $\pi \pi$ scattering lengths are small due to the Goldstone nature of the pions, their final-state rescattering can be taken into account perturbatively, in contrast to what one has to do e.g. in the treatment of three-nucleon systems. Hence in this case, we can make use of a two-fold expansion in $\epsilon$ and $a$, by which we generically denote all $\pi \pi$ threshold parameters. This scheme allows for a consistent power counting in the sense that at any given order in $a$ and $\epsilon$, only a finite number of graphs contributes.

The polynomial terms contributing at tree level are organized in even powers of momenta, hence there are terms of order $\epsilon^{0}, \epsilon^{2}, \epsilon^{4}, \ldots$ Slightly more complicated is the power counting of the loop graphs, of which the typical topologies at one- and two-loop order are shown in Fig. 3. Generically, the non-relativistic pion propagators are of a form

$$
\propto \frac{1}{\omega(\mathbf{p})-p^{0}}=O\left(\epsilon^{-2}\right)
$$

(note however the discussion below on its precise form), while a loop integration is counted according to

$$
d^{4} p=d p^{0} d^{3} \mathbf{p}=O\left(\epsilon^{5}\right) .
$$

Consequently, we find that each additional loop induced by two-body rescattering is suppressed by a factor of

$$
\left(\epsilon^{-2}\right)^{2} \epsilon^{5}=\epsilon,
$$


such that the one-loop diagram (a) in Fig. 3 is of $O\left(a^{1} \epsilon^{1}\right)$, while the two two-loop graphs (b) and (c) are of $O\left(a^{2} \epsilon^{2}\right)$. We therefore find a correlated expansion in $a$ and $\epsilon$ : loops are not only suppressed by powers of the $\pi \pi$ threshold parameters, but in addition by powers of $\epsilon$.

Finally, a rescattering graph due to three-body interactions that first appears at two-loop order, see diagram (d) in Fig. 3, is suppressed by

$$
\left(\epsilon^{-2}\right)^{3}\left(\epsilon^{5}\right)^{2}=\epsilon^{4} .
$$

This diagram is not proportional to any $\pi \pi$ threshold parameters; however, the graph is a constant, and its main effect apart from coupling constant renormalization is to give the $K \rightarrow 3 \pi$ vertex a small imaginary part. Specifically, denoting the leading $O\left(\epsilon^{0}\right) K^{+} \rightarrow \pi^{0} \pi^{0} \pi^{+}$vertex by $G_{0}$ (see Eq. (23) below), the $\pi^{0} \pi^{0} \pi^{+}$intermediate state, with elastic three-particle rescattering approximated by the leading-order vertex derived from chiral perturbation theory, leads to an imaginary part of

$$
\begin{aligned}
\frac{\operatorname{Im} G_{0}}{\operatorname{Re} G_{0}} & =\frac{\left(M_{K}-3 M_{\pi}\right)^{2}}{256 \pi^{2}} \frac{M_{\pi}^{2}}{24 \sqrt{3} F_{\pi}^{4}}+O\left(\left(M_{K}-3 M_{\pi}\right)^{3}\right) \\
& \simeq 1.5 \cdot 10^{-5}
\end{aligned}
$$

which therefore indeed turns out to be formally of $O\left(\epsilon^{4}\right)$, but numerically entirely negligible.

As the cusp effect depends essentially on the analytic properties of the amplitude, it is clearly desirable to preserve the latter exactly, i.e. to correctly reproduce the singularity structure of the relativistic decay amplitude in the low-energy region $|\mathbf{p}| \ll M_{\pi}$; only far-away singularities associated e.g. with the creation and annihilation of particle-antiparticle pairs (inelastic channels) should be subsumed in effective coupling constants. To this end, we use a pion propagator of the form

$$
\frac{1}{2 \omega(\mathbf{p})} \frac{1}{\omega(\mathbf{p})-p^{0}} .
$$

This corresponds to the complete particle-pole piece of the full relativistic propagator,

$$
\frac{1}{M_{\pi}^{2}-p^{2}}=\frac{1}{2 \omega(\mathbf{p})} \frac{1}{\omega(\mathbf{p})-p^{0}}+\frac{1}{2 \omega(\mathbf{p})} \frac{1}{\omega(\mathbf{p})+p^{0}},
$$

and therefore reproduces the correct relativistic dispersion law. The propagator Eq. (20) can be generated by a nonlocal kinetic-energy Lagrangian

$$
\mathcal{L}_{\text {kin }}=\Phi^{\dagger}(2 W)\left(i \partial_{t}-W\right) \Phi, \quad W=\sqrt{M_{\pi}^{2}-\Delta},
$$

where $\Phi$ represents the pion field operator and $\Delta$ is the Laplacian. $\mathcal{L}_{\text {kin }}$ generates all relativistic corrections in the propagator and leads to a manifestly Lorentz-invariant and frame-independent amplitude.

In order to restore the naive power counting rules for loop graphs in the presence of explicit heavy (pion) mass scales, one has to apply the threshold expansion [31,17, 18]: all loop integrals are expanded in powers of the inverse pion mass, integrated order by order, and the results subsequently resummed. In particular the presence of the square roots $\omega(\mathbf{p})$ in the propagator Eq. (20) leads to significant technical complications in the calculation of the loops.

We need two types of interaction terms in the effective Lagrangian, generating the $\pi \pi$ interaction as well as the $K \rightarrow 3 \pi$ tree level amplitudes,

$$
\begin{aligned}
\mathcal{L}_{\pi \pi} & =C_{x}\left(\Phi_{-}^{\dagger} \Phi_{+}^{\dagger}\left(\Phi_{0}\right)^{2}+\text { h.c. }\right)+\ldots+O\left(\epsilon^{2}\right), \\
\mathcal{L}_{K 3 \pi} & =\frac{G_{0}}{2} K_{+}^{\dagger} \Phi_{+}\left(\Phi_{0}\right)^{2}+\frac{H_{0}}{2} K_{+}^{\dagger} \Phi_{-}\left(\Phi_{+}\right)^{2}+\text { h.c. }+O\left(\epsilon^{2}\right),
\end{aligned}
$$

where we have only displayed the leading, energy-independent couplings, and the ellipsis in $\mathcal{L}_{\pi \pi}$ denotes similar interaction terms for the other possible $\pi \pi$ scattering channels. The current accuracy of the calculation of the $K \rightarrow 3 \pi$ decay amplitude includes all terms up to and including $O\left(a^{0} \epsilon^{4}, a^{1} \epsilon^{5}, a^{2} \epsilon^{4}\right)$; for this purpose, $\mathcal{L}_{K 3 \pi}$ as well as $\mathrm{S}$-wave interaction terms in $\mathcal{L}_{\pi \pi}$ are needed up to $O\left(\epsilon^{4}\right)$, while only P-wave scattering lengths and no D-wave contributions are necessary in $\mathcal{L}_{\pi \pi}$.

The whole framework briefly sketched here is a completely Lagrangian-based quantum field theory, hence all constraints from analyticity and unitarity are automatically obeyed.

\subsection{Matching}

The coupling constants of the non-relativistic Lagrangians Eq. (23) have to be related to physical observables in the underlying relativistic field theory. In the case of the couplings of $\mathcal{L}_{\pi \pi}$, they can be matched using the effective range expansion of the $\pi \pi$ scattering amplitude. For example, $C_{x}$ as defined in Eq. (23) is related to the chargeexchange amplitude $T_{x}=T\left(\pi^{+} \pi^{-} \rightarrow \pi^{0} \pi^{0}\right)$ by

$$
\begin{aligned}
\operatorname{Re} T_{x}= & 2 C_{x}+O\left(\epsilon^{2}\right) \\
2 C_{x}= & -\frac{32 \pi}{3}\left(a_{0}^{0}-a_{0}^{2}\right)\left\{1+\frac{M_{\pi^{+}}^{2}-M_{\pi^{0}}^{2}}{3 M_{\pi}^{2}}\right\}+O\left(e^{2} p^{2}\right) \\
= & -\frac{32 \pi}{3}\left(a_{0}^{0}-a_{0}^{2}\right)\left\{1+(0.61 \pm 0.16) \times 10^{-2}\right\} \\
& +O\left(e^{2} p^{4}\right) .
\end{aligned}
$$

The isospin-breaking corrections in relating the chargeexchange amplitude at threshold to the scattering lengths of definite isospin are calculated in chiral perturbation theory, where the second line in Eq. (24) shows the analytic correction at $O\left(e^{2}\right)$, while the numerical estimate in the third line includes the higher order of $O\left(e^{2} p^{2}\right)$ [32,17].

The polynomial terms $G_{0}, G_{1}, \ldots$ for $K^{+} \rightarrow \pi^{0} \pi^{0} \pi^{+}$ and $H_{0}, H_{1}, \ldots$ for $K^{+} \rightarrow \pi^{+} \pi^{+} \pi^{-}$are not strictly matched, but used as a parameterization of the amplitudes in question. They replace the more traditional Dalitz plot parameters used for that purpose in experimental fits neglecting non-trivial final-state rescattering effects. The strategy is to fit (in principle) all parameters of the non-relativistic representation to data of both decay channels, and then determine the scattering length combination $a_{0}^{0}-a_{0}^{2}$ via Eq. (24). 
In practice, one may decide to use some of the parameters, for instance higher-order $\pi \pi$ threshold parameters such as effective ranges or P-waves, as input, employing their theoretically predicted values [6] instead.

\subsection{Analytic structure of the non-trivial two-loop graph}

The function $F(s)$ describing the non-trivial, genuine twoloop graph (c) in Fig. 3 can be expressed analytically in terms of logarithms (see Ref. [33] for an explicit closed representation). Close to threshold, it can be approximated according to

$$
F(s) \simeq \frac{v_{ \pm}(s)}{256 \pi^{2}} \sqrt{\frac{M_{K}^{2}-9 M_{\pi}^{2}}{M_{K}^{2}-M_{\pi}^{2}}}
$$

(for all pion masses running in the loop equal), which is manifestly of $O\left(\epsilon^{2}\right)$ as required by the power counting set up in Sect. 3.1. However, a decomposition of the full twoloop function according to

$$
F(s)=A(s)+B(s) v_{ \pm}(s),
$$

with both $A(s), B(s)$ analytic functions of $s$ as suggested in Ref. [28], turns out not to hold. In fact, it can be shown [34] that if one enforces such a decomposition, both $A(s)$ and $B(s)$ diverge at the border of phase space for maximal $s$ like $1 / \sqrt{s_{p}-s}, s_{p}=\left(M_{K}-M_{3}\right)^{2}$, in such a way that the sum $A(s)+B(s) v_{ \pm}(s)$ is finite.

What is more, at least for certain pion mass assignments within the loop, the decomposition Eq. (26) even fails as a representation of the analytic structure of $F(s)$ within the decay region. With the pions in the loop labelled as indicated in Fig. 3, the solutions of the Landau equations $[35,36]$ show that anomalous thresholds exist for

$$
\begin{aligned}
& s^{ \pm}= \frac{1}{2}\left\{M_{K}^{2}+M_{3}^{2}+M_{c}^{2}+M_{d}^{2}-\left(M_{a}+M_{b}\right)^{2}\right. \\
&\left.+\frac{\left(M_{K}^{2}-M_{c}^{2}\right)\left(M_{d}^{2}-M_{3}^{2}\right) \pm \sqrt{\lambda_{1} \lambda_{2}}}{\left(M_{a}+M_{b}\right)^{2}}\right\}, \\
& \lambda_{1}=\lambda\left(\left(M_{a}+M_{b}\right)^{2}, M_{3}^{2}, M_{d}^{2}\right), \\
& \lambda_{2}=\lambda\left(M_{K}^{2},\left(M_{a}+M_{b}\right)^{2}, M_{c}^{2}\right),
\end{aligned}
$$

with the standard Källén function $\lambda(a, b, c)=a^{2}+b^{2}+c^{2}-$ $2(a b+a c+b c)$. According to Eq. (27), the analytic structure of $F(s)$ is particularly intricate for $M_{a}+M_{b} \neq M_{3}+M_{d}$. The two relevant graphs in $K^{+} \rightarrow \pi^{0} \pi^{0} \pi^{+}$fulfilling this condition are shown in Fig. 4. In the case of diagram (a), the $s_{1}^{ \pm}$are real and yield branch points in the amplitude at $\sqrt{s_{1}^{-}}=308 \mathrm{MeV}$ and $\sqrt{s_{1}^{+}}=356 \mathrm{MeV}$, compared to the phase space limits in this variable, given by threshold $\sqrt{s_{t}}=275 \mathrm{MeV}$ and pseudothreshold $\sqrt{s_{p}}=359 \mathrm{MeV}$. The functions $A(s)$ and $B(s)$ in the decomposition Eq. (26) display singular behavior at $s_{1}^{ \pm}$, while the complete amplitude is analytic at the upper rim of the cut (that starts at $\left.s_{t}=\left(M_{\pi^{+}}+M_{\pi^{0}}\right)^{2}\right)$; it becomes singular only at its lower

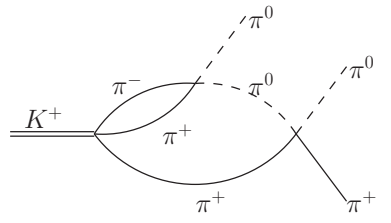

(a)

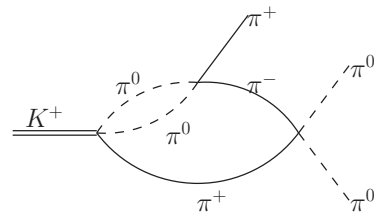

(b)
Fig. 4. Two-loop diagrams for $K^{+} \rightarrow \pi^{0} \pi^{0} \pi^{+}$displaying anomalous thresholds. Double, single, and dashed lines stand for charged kaons, charged, and neutral pions, respectively.

rim. Diagram (b), on the other hand, has complex anomalous thresholds $s_{3}^{ \pm}$, which lead to special complications in a dispersive representation of this loop graph, necessitating a deformation of the integration path over the discontinuity. Again, on the upper rim of the cut, the complete loop function $F(s)$ is analytic also in that case.

We wish to emphasize once more that the singularity structure of the explicit non-relativistic representation of $F(s)[33]$ in the low-energy region, i.e. in the decay region and slightly beyond, is identical to the one of the fully relativistic amplitude. It therefore includes all the anomalous thresholds discussed above, in precisely the right kinematical positions.

\subsection{Two-loop representation}

The full representation of the $K \rightarrow 3 \pi$ decay amplitudes up to and including $O\left(a^{0} \epsilon^{4}, a^{1} \epsilon^{5}, a^{2} \epsilon^{4}\right)$ comprises tree, oneloop, and two-loop graphs of the topologies shown in Fig. 3, with all possible charge combinations of intermediate pions. The only loop function at one loop, see graph (a) in Fig. 3, is given by

$$
J(s)=\frac{i v(s)}{16 \pi},
$$

hence produces precisely the analytic structure discussed in Sect. 2. The two-loop graph (b) in Fig. 3 is given as a product of two functions $J(s)$, hence it is real above threshold and, if it contains singular behavior at $s_{3}=4 M_{\pi^{+}}^{2}$ (in a product of one "charged" and one "neutral" loop), the real square root that interferes with the dominant tree graphs is also seen for $s_{3}>4 M_{\pi^{+}}^{2}$. The same is true for the threshold behavior of the genuine two-loop function discussed in the last section, see Eq. (25). Finally, the two-loop graph with three-body rescattering, diagram (d) in Fig. 3, is a constant and can essentially be absorbed in a redefinition of the treelevel couplings, hence it does not affect the analytic structure in a non-trivial way.

The cusp up to two loops is therefore of the following generic structure: while the one-loop diagrams generate a structure $\propto$ i $a v_{ \pm}\left(s_{3}\right)$ which interferes with the (dominant) tree amplitude below the $\pi^{+} \pi^{-}$threshold (where the square root turns real), the two-loop graphs include terms $\propto a^{2} v_{ \pm}\left(s_{3}\right)$, hence a singular structure (in interference with the tree parts) above that point. This is illustrated schematically in Fig. 5. The "two-loop cusp" above threshold is a 

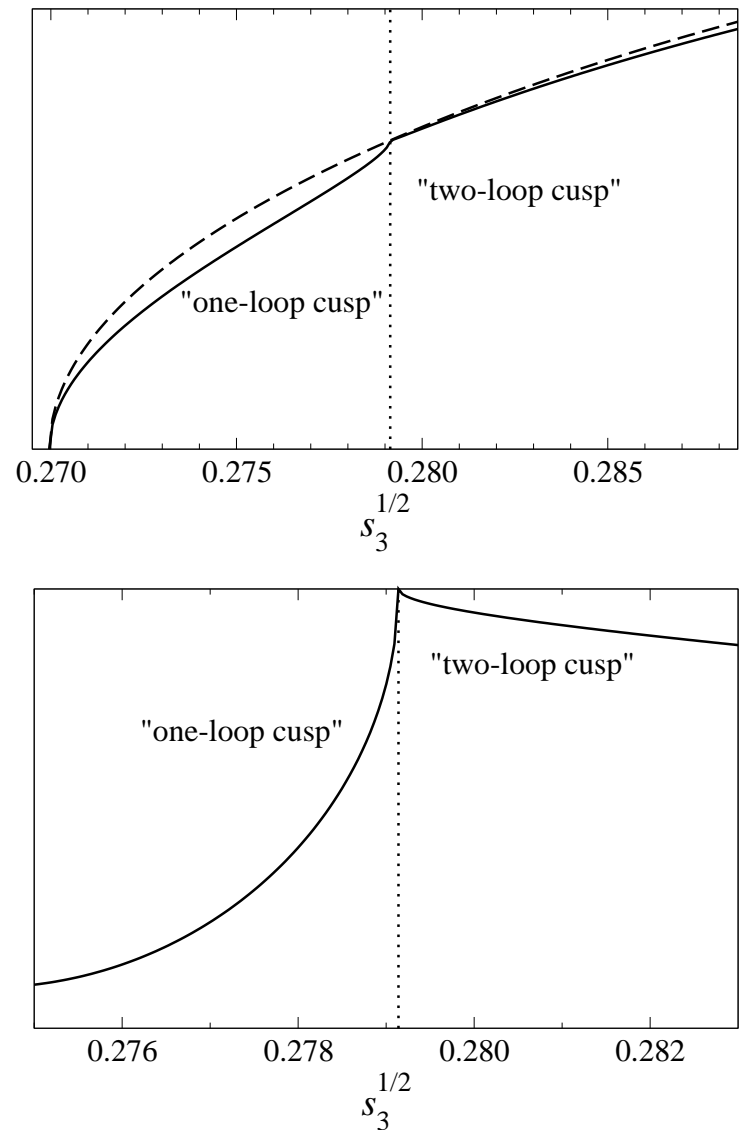

Fig. 5. Sketch of the cusps in the decay spectrum at $O(a)$ below, and at $O\left(a^{2}\right)$ above the $\pi^{+} \pi^{-}$threshold, denoted by the vertical dotted line. In the lower panel, focussing even closer on the threshold region, the tree-level spectrum (dashed) is subtracted for better illustration of the small "two-loop cusp".

much smaller effect, yet given the precision of the data in the NA48/2 analysis, it has turned out to be a vital ingredient in the theoretical representation in order to achieve a statistically adequate description. As the cusp strength at two loops also incorporates $\pi \pi$ rescattering effects other than the charge exchange channel, there is in principle also (reduced) sensitivity to another linear combination of Swave scattering lengths, e.g. $a_{0}^{2}$ alone. A fit of this form yields [27]

$$
\begin{aligned}
a_{0}^{0}-a_{0}^{2} & =0.2815 \pm 0.0043_{\text {stat }} \pm \ldots, \\
a_{0}^{2} & =-0.0693 \pm 0.0136_{\text {stat }} \pm \ldots .
\end{aligned}
$$

A comparison to the theoretical prediction Eq. (6) shows that $a_{0}^{0}-a_{0}^{2}$ comes out uncomfortably large (by more than $3.5 \sigma)$. This turns out not to be a statistical accident, but there is a theoretical explanation for this discrepancy that we will discuss in the following section.

\section{Radiative corrections}

Once the theoretical and experimental precision in the determination of hadronic, strong-interaction physics observ-

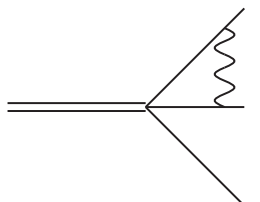

(a)

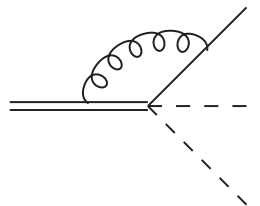

(b)

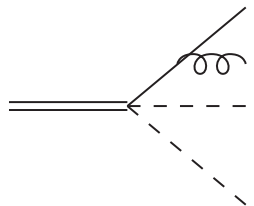

(c)
Fig. 6. Examples for diagrams with "external" radiative corrections. Double, single, and dashed lines stand for charged kaons, charged, and neutral pions, respectively. Wiggly and curly lines denote Coulomb and transverse photons, respectively.

ables arrives at the percent level, the effects of electromagnetic or radiative corrections have to be taken into account. Such corrections in $K \rightarrow 3 \pi$ decays have already been considered earlier in the framework of chiral perturbation theory [37-39], or in a quantum-mechanical approach [40, 41]. As we employ a Lagrangian framework, the inclusion of photons via minimal substitution is completely straightforward:

$$
\partial_{\mu} \Phi_{ \pm} \rightarrow\left(\partial_{\mu} \mp i e A_{\mu}\right) \Phi_{ \pm}
$$

and similarly for the kaons. Furthermore, all possible nonminimal gauge invariant terms can be added. In the context of a non-relativistic theory, it is useful to work in the Coulomb gauge and differentiate between Coulomb and transverse photons, which feature differently in the generalized power counting scheme. In addition, for transverse photons, one has to differentiate between "soft" and "ultrasoft" modes: while both have zero components that have to be counted according to $l_{0}=O\left(\epsilon^{2}\right)$, the three components are either $\mathbf{I}=O(\epsilon)$ for soft, or $\mathbf{I}=O\left(\epsilon^{2}\right)$ for ultrasoft photons. The summary of the counting rules for diagrams with virtual photons is then as follows [42]:

1. Adding a Coulomb photon to a hadronic "skeleton" diagram modifies its counting by a factor of $e^{2} / \epsilon$. An example of this is diagram (a) in Fig. 6: with a constant $K \rightarrow 3 \pi$ vertex of $O\left(\epsilon^{0}\right)$, this diagram will scale as $O\left(e^{2} \epsilon^{-1}\right)$, the negative power in $\epsilon$ indicating the presence of the Coulomb pole in that graph.

2 . Transverse photons couple to mesons with vertices of $O(\epsilon)$, hence soft transverse photons are suppressed relative to Coulomb photon exchange by two orders in the $\epsilon$-expansion. As an example, diagram (a) in Fig. 6, with the Coulomb photon replaced by a transverse one, contributes at $O\left(e^{2} \epsilon\right)$.

3. Ultrasoft transverse photons added to a hadronic "skeleton" diagram finally change its power counting by a factor of $e^{2} \epsilon^{2}$. As an example, the transverse photon in diagram (b) of Fig. 6 can be shown to be ultrasoft, hence with a constant $K \rightarrow 3 \pi$ vertex, the graph scales as $O\left(e^{2} \epsilon^{2}\right)$.

As is well known, the inclusion of virtual photon effects requires the simultaneous consideration of radiation of additional real photons in order to obtain well-defined, infrared-finite quantities. The observable that can be calculated including effects of $O(\alpha)$ (where $\alpha=e^{2} / 4 \pi$ is the 

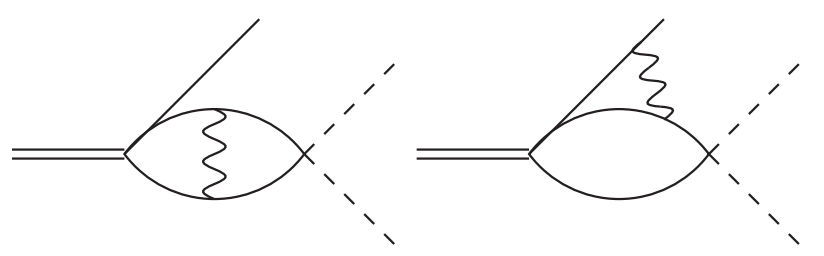

Fig. 7. Examples for diagrams with "internal" radiative corrections. The wiggly lines denote Coulomb photons; otherwise, see the line style in Fig. 6.

fine structure constant) is [42]

$$
\begin{aligned}
\left.\frac{d \Gamma}{d s_{3}}\right|_{E_{\gamma}<E_{\max }} & =\frac{d \Gamma(K \rightarrow 3 \pi)}{d s_{3}}+\left.\frac{d \Gamma(K \rightarrow 3 \pi \gamma)}{d s_{3}}\right|_{E_{\gamma}<E_{\max }}+O\left(\alpha^{2}\right) \\
& =\Omega\left(s_{3}, E_{\max }\right) \frac{d \Gamma^{\mathrm{int}}}{d s_{3}}+O\left(\alpha^{2}\right) .
\end{aligned}
$$

The notation indicates that the emission of real or bremsstrahlung photons is included up to a maximal energy $E_{\max }$, specified by the experimental detector resolution. Here, the channel-dependent correction factor $\Omega\left(s_{3}, E_{\max }\right)$ subsumes all "external" radiative corrections due to (real and virtual) corrections with the photons exclusively hooked to charged external legs, see Fig. 6 for examples, while $d \Gamma^{\mathrm{int}} / d s_{3}$ still includes other, "internal" radiative corrections, see Fig. 7. In terms of the non-relativistic power counting, we aim for a calculation of the decay spectra Eq. (31) including effects of $O\left(e^{2} \epsilon^{4}\right)$ for all $K \rightarrow 3 \pi$ channels. One has to bear in mind that the non-radiative decay spectrum $d \Gamma(K \rightarrow 3 \pi) / d s_{3}$ starts at $O\left(\epsilon^{2}\right)$, while the bremsstrahlung part $d \Gamma(K \rightarrow 3 \pi \gamma) / d s_{3}$ begins to contribute at $O\left(e^{2} \epsilon^{4}\right)$, which means that it is sufficient to include radiation of real photons at leading order in the nonrelativistic expansion. Internal corrections of $O\left(e^{2} a^{1} \epsilon^{2}\right)$, on the other hand, are only included for the "main" decay channels displaying the cusp, $K^{+} \rightarrow \pi^{0} \pi^{0} \pi^{+}$and $K_{L} \rightarrow 3 \pi^{0}$ (see Sect. 6.1 below).

The external radiative corrections are rather wellknown (compare e.g. Ref. [43] for a relativistic approach); their effect on the decay spectrum Eq. (31) is small and smooth except for the Coulomb pole in channels with more than one charged particle in the final state. These latter threshold singularities are usually taken care of by means of the Gamow-Sommerfeld factors $[44,45]$ in the experimental analyses. Even the soft-photon approximation for bremsstrahlung photons is rather accurate compared to the exact result [42]. In fact, the non-relativistic power counting and in particular the resulting powers in $\epsilon$ for the various radiative corrections nicely illustrate why the effects of Coulomb photons in particular are important, while (finite) bremsstrahlung effects are very small.

In the context of the cusp analysis, the internal corrections are of potentially more intriguing effect, as they modify the analytic structure of the decay amplitude near the $\pi^{+} \pi^{-}$threshold. These modifications become important as soon as $\alpha / v_{ \pm}$is not small any more. The most remarkable effect is the formation of pionium due to multi-photon exchange inside the charged-pionium loop, see Fig. 8, which

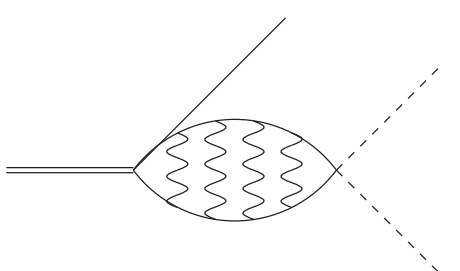

Fig. 8. Multi-photon exchange inside the charged-pion loop, responsible for pionium formation.

leads to an infinite number of bound-state poles close to threshold. The analytic solution to the resummation of an infinite number of exchanged Coulomb photons is known as the Schwinger Green's function [46] replacing the simple one-loop function $J_{ \pm}(s)$ in Eq. (28),

$$
\begin{aligned}
G(s) & =\frac{i v_{ \pm}(s)}{16 \pi} \\
& -\frac{\alpha}{16 \pi}\left[\frac{\log \left(-v_{ \pm}^{2}(s)\right)}{2}+\Psi\left(1-\frac{i \alpha}{2 v_{ \pm}(s)}\right)-\Psi(1)+C\right]
\end{aligned}
$$

where $\Psi(x)=\frac{d}{d x} \log \Gamma(x)$, and $C$ is a constant. The function $\Psi$ contains the pionium pole terms, which are nonperturbative effects, with binding energies of $O\left(\alpha^{2}\right)$. The leading effect however is, at $O(\alpha)$, the one-photon exchange, leading to the logarithmic divergence at threshold $\propto \log \left(-v_{ \pm}^{2}(s)\right)$ in Eq. (32). Even if the central bin right at the cusp where pionium is formed is excluded in the experimental analysis, such that the one-photon exchange is sufficient to describe the effects of electromagnetism, its effects are surprisingly sizeable. This is due to the fact that the cusp is precisely about a change in the slope of the distribution: if its structure below threshold is, up to $O(\alpha)$, given by

$$
-\frac{1}{16 \pi^{2}}\left\{\sqrt{-v_{ \pm}^{2}(s)}+\frac{\alpha}{2} \log \left(-v_{ \pm}^{2}(s)\right)\right\},
$$

its derivative at a kinematical point $s=4 M_{\pi^{+}}^{2}-\Delta$ yields

$$
\frac{1}{64 \pi M_{\pi} \sqrt{\Delta}}\left\{1+\frac{2 \alpha M_{\pi}}{\sqrt{\Delta}}\right\}+\ldots,
$$

where the ellipsis denotes higher orders in $\Delta$, such that the correction of $O(\alpha)$ becomes large near threshold. Indeed, the fit to experimental data, including radiative corrections in the amplitude, yields the following results for the $\pi \pi$ scattering lengths [27]:

$$
\begin{aligned}
a_{0}^{0}-a_{0}^{2} & =0.2571 \pm 0.0048_{\text {stat }} \pm 0.0025_{\text {syst }} \pm 0.0014_{\text {ext }}, \\
a_{0}^{2} & =-0.024 \pm 0.013_{\text {stat }} \pm 0.009_{\text {syst }} \pm 0.002_{\text {ext }} \text {. }
\end{aligned}
$$

Comparing Eq. (35) to Eq. (29), we see that radiative corrections decrease the extracted central value for $a_{0}^{0}-a_{0}^{2}$ by nearly $10 \%$, so their inclusion turns out to be absolutely essential at this accuracy.

In Fig. 9, we show the combined experimental results on the S-wave $\pi \pi$ scattering lengths from modern precision determinations. Shown are the ellipse (full) extracted from 


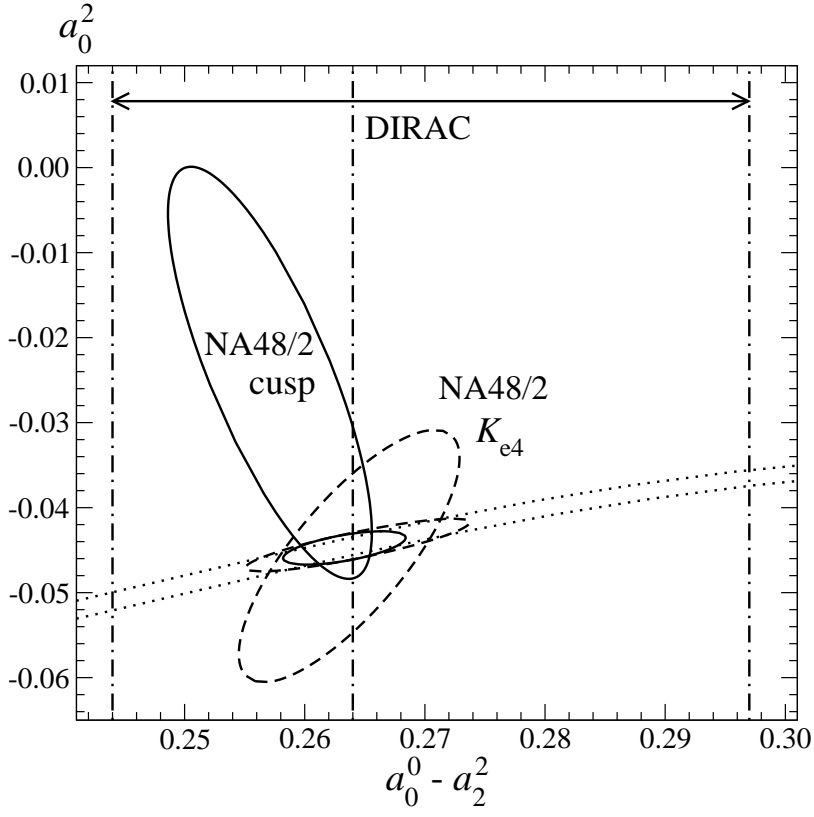

Fig. 9. Combined experimental results on the S-wave $\pi \pi$ scattering lengths from the pionium width as obtained by the DIRAC collaboration [12], $K_{e 4}$ [14], and the cusp in $K^{ \pm} \rightarrow \pi^{0} \pi^{0} \pi^{ \pm}$[27], the latter two as measured by the NA48/2 collaboration. For details, see text. Data obtained from Ref. [27].

the cusp analysis in $K^{ \pm} \rightarrow \pi^{0} \pi^{0} \pi^{ \pm}$described here [27], the NA48/2 results from $K_{e 4}$ decays [14] (dashed) using theoretical input on isospin-breaking corrections [21], and the constraint from the pionium lifetime obtained by the DIRAC experiment [12] as the vertical dash-dotted band. The narrow dotted band shows the correlation between $a_{0}^{0}$ and $a_{0}^{2}$ dictated by the relation of $\bar{\ell}_{4}$ to the scalar radius of the pion [6], the smaller ellipses are fits to the two NA48/2 experiments using the chiral perturbation theory constraint. Altogether, a most impressive agreement between the different experiments as well as experiment and theory has been achieved.

\section{On the accuracy of the extraction$$
\text { of } a_{0}^{0}-a_{0}^{2}
$$

An important ingredient yet missing to finally assess the accuracy of the extracted values for the $\pi \pi$ scattering lengths is a reliable estimate of the theoretical uncertainty inherent in the representation of the amplitude. In the first publication of an experimental cusp analysis [22], a generic theoretical error of 5\% was assumed, following a suggestion made in Ref. [28], thus the theoretical input dominated the final uncertainty. The following main points may be responsible for the theoretical error.

1. Radiative corrections. These are now taken care of [42], we regard the remaining uncertainty from higher-order radiative corrections as entirely negligible.
2. Isospin-breaking corrections in the matching relations. These are small, the uncertainty is estimated to be $\lesssim$ $1 \%$, see Eq. (24).

3. The effects of higher-order derivative interactions in the two-loop contributions, i.e. terms of $O\left(a^{2} \epsilon^{4}\right)$ and higher. Their impact is still under investigation [34], although there are indications that the scattering lengths are very stable under such modifications of the amplitude.

4. Higher loop contributions, starting at three loops $O\left(a^{3} \epsilon^{3}\right)$.

For this last point, we wish to discuss the so-called threshold theorem [26,23,30,42] (valid in the absence of photons). It states that the coefficient of the leading cusp behavior (or $v_{ \pm}\left(s_{3}\right)$ ) is proportional to the product

$$
\left.T\left(K^{+} \rightarrow \pi^{+} \pi^{+} \pi^{-}\right)\right|_{\mathrm{thr}} \times\left. T\left(\pi^{+} \pi^{-} \rightarrow \pi^{0} \pi^{0}\right)\right|_{\mathrm{thr}},
$$

where the second factor is just the combination of scattering lengths given in Eq. (24), and the "threshold" at which the first factor is to be evaluated refers to $s_{1}=4 M_{\pi^{+}}^{2}, s_{2}=$ $s_{3}=\left(M_{K}^{2}-M_{\pi^{+}}^{2}\right) / 2$. In other words, knowing the decay amplitude for the charged final state $T\left(K^{+} \rightarrow \pi^{+} \pi^{+} \pi^{-}\right)$to $O\left(a^{n}\right)$ allows one to determine the dominant cusp strength of $T\left(K^{+} \rightarrow \pi^{0} \pi^{0} \pi^{+}\right)$at $O\left(a^{n+1}\right)$. As we have the full twoloop representation available for all $K \rightarrow 3 \pi$ channels, we can estimate the size of the cusp at three loops by the expansion

$\left.T\left(K^{+} \rightarrow \pi^{+} \pi^{+} \pi^{-}\right)\right|_{\mathrm{thr}} \propto-1.0_{\text {tree }}-0.13 i_{1-\text { loop }}+0.014_{2-\text { loop }}$,

which suggests that the three-loop cusp will modify the leading (one-loop) effect by about $1.5 \%$. This estimate is no substitute for a complete three-loop calculation, as it does not yield a representation of $O\left(a^{3}\right)$ elsewhere in the decay region except near the cusp, and neither does it contain information about subleading non-analytic behavior near threshold (e.g. $\propto v_{ \pm}^{3}\left(s_{3}\right)$ ). Still, we regard Eq. (37) as a good indication for the rate of convergence in the $K^{+} \rightarrow \pi^{0} \pi^{0} \pi^{+}$amplitude.

\section{Cusps in other decays}

$6.1 K_{L} \rightarrow 3 \pi^{0}, \eta \rightarrow 3 \pi^{0}$

The mechanism generating the cusp in the $\pi^{0} \pi^{0}$ invariant mass distribution is rather generic and only due to the finalstate interactions between the pions. We may therefore anticipate that other decays into two neutral pions plus a third particle will show a very similar cusp effect, the most obvious examples being $K_{L} \rightarrow 3 \pi^{0}$ and $\eta \rightarrow 3 \pi^{0}$. The effect of the cusp in these channels has been investigated theoretically $[28,29,33,47,48]$, and first efforts to see it experimentally have been reported both for $K_{L} \rightarrow 3 \pi^{0}$ [49] and $\eta \rightarrow 3 \pi^{0}$ [50-52] decays. The main difference between these and $K^{+} \rightarrow \pi^{0} \pi^{0} \pi^{+}$, however, is the following. As indicated in Fig. 10, the extent to which the decay spectrum with respect to the invariant mass squared of the $\pi^{0} \pi^{0}$ pair is perturbed by the cusp effect does not only depend 


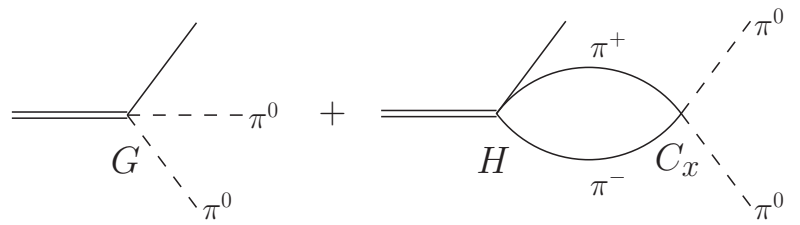

Fig. 10. Mechanism for cusp effects in generic decays into three final-state hadrons, including two $\pi^{0}$.

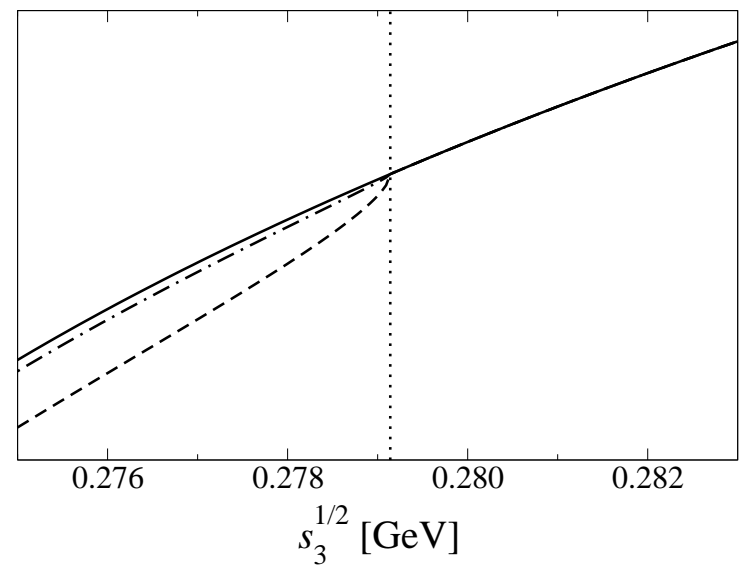

Fig. 11. Sketches of the leading (one-loop) cusp effects on the decay spectra for $K^{+} \rightarrow \pi^{0} \pi^{0} \pi^{+}$(dashed line) and $K_{L} \rightarrow 3 \pi^{0}$ (dash-dotted line) in the vicinity of the $\pi^{+} \pi^{-}$threshold, marked by the dotted vertical line. The full line denotes the unperturbed spectrum without $\pi \pi$ rescattering.

on the charge-exchange scattering length as encoded in the coupling constant $C_{x}$, but strictly speaking, it is rather proportional to

$$
\frac{H}{G} \times C_{x},
$$

where $G$ and $H$ generically denote the coupling strengths to the "neutral" and "charged" final state, i.e. $\pi^{0} \pi^{0}$ and $\pi^{+} \pi^{-}$plus a third meson, respectively. In other words, the strength of the cusp depends crucially on the relative branching fractions into the charged and neutral final states: the more the decay into charged pions is preferred, the better the magnification of the effect in the spectrum.

It turns out that the ratio $|H / G|$ is very different for the different decays with three-pion final states. While for the $K^{+}$decays considered so far, it is approximately 2, both for $K_{L}$ and $\eta$ it is closer to $1 / 3$, in other words the $K_{L}$ and $\eta$ prefer to decay into $3 \pi^{0}$. To illustrate the difference, we sketch the leading (one-loop, $O(a)$ ) cusps for $K^{+} \rightarrow \pi^{0} \pi^{0} \pi^{+}$and $K_{L} \rightarrow 3 \pi^{0}$ (the picture for $\eta \rightarrow 3 \pi^{0}$ is very similar to the latter case) in Fig. 11. While the square-root-like structure is clearly visible to the naked eye for the $K^{+}$decay, it is much harder to discern in the case of the $K_{L}$. For this reason, it is also much harder experimentally to achieve a precision determination of $\pi \pi$ scattering lengths from an investigation of the cusps in $K_{L} \rightarrow 3 \pi^{0}$ or $\eta \rightarrow 3 \pi^{0}$. More quantitatively, while the cusp effect reduces the number of events below the $\pi^{+} \pi^{-}$threshold in the $K^{+} \rightarrow \pi^{0} \pi^{0} \pi^{+}$spectrum by about $13 \%$ [53], for e.g. $\eta \rightarrow 3 \pi^{0}$, the corresponding reduction amounts to only $1-2 \%$ [48]. So while prob- ably no competitive scattering length determination from these channels seems feasible in the near future, the cusp effect should at least be taken into account in ongoing or future precision determinations of the $\eta \rightarrow 3 \pi^{0}$ Dalitz plot slope parameter $\alpha$ [50-52]; compare also Ref. [47].

\section{$6.2 \eta^{\prime} \rightarrow \eta \pi^{0} \pi^{0}$}

At least from a theoretical perspective, much more promising in this respect is the decay $\eta^{\prime} \rightarrow \eta \pi^{0} \pi^{0}$ [54]. With the $\eta^{\prime}$ and the $\eta$ both being particles of isospin 0 , it is obvious that in the approximation of isospin conservation, the $\pi \pi$ pair is produced with total isospin 0 , which immediately shows that the amplitude for $\eta^{\prime} \rightarrow \eta \pi^{+} \pi^{-}$is enhanced compared to the $\eta^{\prime} \rightarrow \eta \pi^{0} \pi^{0}$ one by a factor of $-\sqrt{2}$ (the sign is according to the Condon-Shortley phase convention). We therefore expect a cusp much more prominent than in $K_{L}, \eta \rightarrow 3 \pi^{0}$, if not quite as pronounced as in $K^{+} \rightarrow \pi^{0} \pi^{0} \pi^{+}$. From the experimental perspective, the upcoming high-statistics $\eta^{\prime}$ experiments at ELSA [55], MAMI-C [56-58], WASA-at-COSY [59,60], KLOE-atDA $\Phi$ NE [61,62], or BES-III [63] are expected to increase the data basis on $\eta^{\prime}$ decays by orders of magnitude, so an investigation of the cusp effect in this channel seems very promising.

What makes this channel somewhat different from those investigated so far is the presence of the $\eta$ in the final state, and hence of $\pi \eta$ rescattering as a new ingredient to final-state interactions. There is no experimental information on $\pi \eta$ threshold parameters, and it turns out that chiral symmetry constrains these quantities only very badly [64, 65]: the $O\left(p^{4}\right)$ corrections to the current algebra prediction of the S-wave scattering length, for example, can easily be as big as or bigger than the leading order. The one thing that chiral perturbation theory does seem to predict reliably is the fact that $\pi \eta$ threshold parameters are systematically smaller than the $\pi \pi$ ones. In conventions comparable to those chosen in $\pi \pi$ scattering (see Ref. [54] for details), the S-wave $\pi \eta$ scattering length is given at leading order by

$$
\bar{a}_{0}=\frac{M_{\pi}^{2}}{96 \pi F_{\pi}^{2}}+O\left(M_{\pi}^{4}\right),
$$

which compared to $a_{0}^{0}$, see Eq. (4), is smaller by a factor of 21 . We therefore expect the effect of the $\pi \eta$ final-state interactions to be significantly smaller than that of $\pi \pi$ rescattering.

For an investigation of the impact of the $\pi \eta$ threshold parameters on the cusp effect in $\eta^{\prime} \rightarrow \eta \pi^{0} \pi^{0}$, we vary them in a sensible range, suggested by various sets of next-toleading order low-energy constants. In Fig. 12, we show the decay spectrum for $\eta^{\prime} \rightarrow \eta \pi^{0} \pi^{0}$ with respect to the invariant mass of the $\pi^{0} \pi^{0}$ pair, comparing the spectrum calculated from the tree-level amplitude to that given by the full two-loop result. The tree-level couplings are fixed by (the central values of) the Dalitz plot parameters determined in Ref. [66], and we assume isospin symmetry between these couplings for the $\eta^{\prime} \rightarrow \eta \pi^{0} \pi^{0}$ and $\eta^{\prime} \rightarrow \eta \pi^{+} \pi^{-}$ channels. There is a very clear signal of the cusp effect 


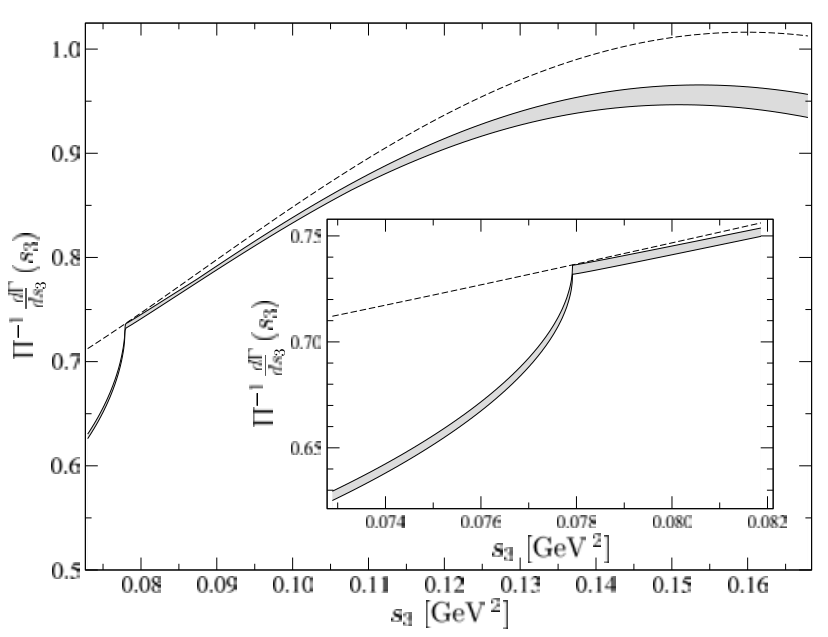

Fig. 12. Decay spectrum for $\eta^{\prime} \rightarrow \eta \pi^{0} \pi^{0}$, divided by pure phase space. The insert focuses on the cusp region around the $\pi^{+} \pi^{-}$ threshold. The dashed line corresponds to the tree result, the gray band shows the full result to two loops, with the uncertainty due to the variation of the $\pi \eta$ threshold parameters. Figure taken from Ref. [54].

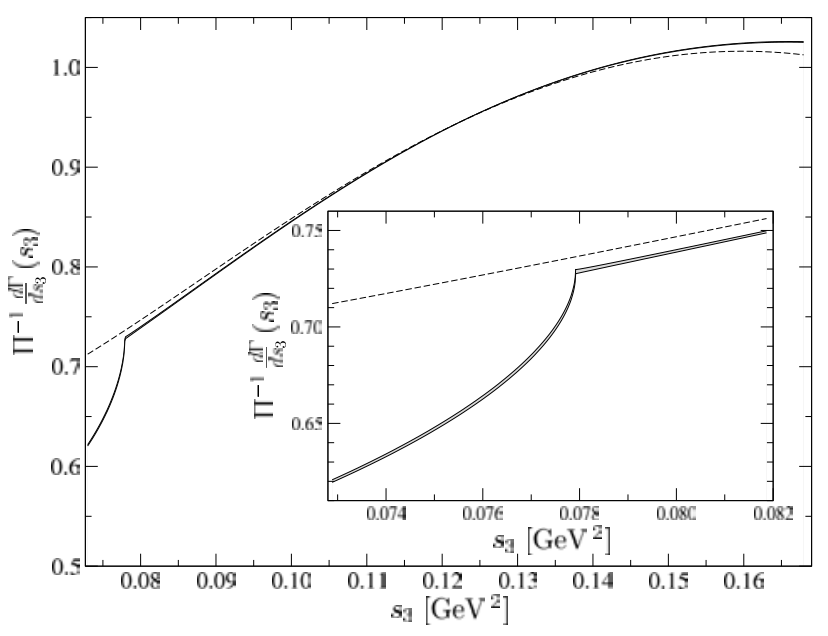

Fig. 13. Decay spectrum for $\eta^{\prime} \rightarrow \eta \pi^{0} \pi^{0}$ as in Fig. 12, after renormalization of tree couplings in order to reproduce the Dalitz plot parameters from Ref. [66] with the full amplitude. Figure taken from Ref. [54].

below the $\pi^{+} \pi^{-}$threshold, plus a significant deviation between tree and two-loop spectrum mainly due to $\pi \pi$ final state interactions at large $s_{3}=M_{\pi^{0} \pi^{0}}^{2}$. The width of the band gives an indication of the size of $\pi \eta$ rescattering effects, which however hardly affect the cusp region.

Obviously, the Dalitz plot parameters of the distribution including loop corrections in Fig. 12 are not identical to the input parameters any more. In Fig. 13, we have therefore renormalized the tree-level couplings in such a way that the full amplitude reproduces the Dalitz plot expansion as measured in Ref. [66]. The result is very striking, as the by far largest part of the final-state interactions above the $\pi^{+} \pi^{-}$threshold can be absorbed into such a redefinition of the tree-level parameters. In particular, hardly any effect of the variation of $\pi \eta$ threshold parameters is visible any more. On the other hand, the prediction of the cusp seems extremely stable.

A remarkable feature of Figs. 12, 13 is the fact that there seems to be hardly any trace left of what we discussed as the "two-loop cusp" in $K^{+} \rightarrow \pi^{0} \pi^{0} \pi^{+}$decays, i.e. a square-root-like behavior above the $\pi^{+} \pi^{-}$threshold. This observation bears up against closer scrutiny: numerically we find that the cusp above threshold in $\eta^{\prime} \rightarrow \eta \pi^{0} \pi^{0}$ is suppressed by about a factor of 250 compared to the leading, $O(a)$ cusp. The explanation for this suppression can be found with the help of the threshold theorem again, see Sect. 5, and it turns out to be the result of residual approximate isospin symmetry between the amplitudes for $\eta^{\prime} \rightarrow \eta \pi^{0} \pi^{0}$ and $\eta^{\prime} \rightarrow \eta \pi^{+} \pi^{-}$[54]. So even if we allowed for small isospin breaking in the tree-level couplings (which was neglected here), a strong relative suppression would persist.

Finally, we can estimate the size of a potential threeloop cusp in analogy to Sect. 5, which turns out not to be suppressed by similar arguments, although, naturally, by the high power of scattering lengths involved. In this case, we find that the cusp of $O\left(a^{3}\right)$ should reduce the leading $O(a)$ cusp by about $0.5 \%$ [54]. So in contrast to $K^{+} \rightarrow$ $\pi^{0} \pi^{0} \pi^{+}$decays, for the description of which the $O\left(a^{2}\right)$ cusp turned out to be absolutely necessary, in the case of $\eta^{\prime} \rightarrow$ $\eta \pi^{0} \pi^{0}$ the singularity is entirely dominated by the leading, one-loop rescattering term.

\subsection{The role of $\pi \eta$ interactions in $\eta^{\prime} \rightarrow \eta \pi^{0} \pi^{0}$}

The cusp effect in the $\pi^{0} \pi^{0}$ invariant mass spectrum of the decay $\eta^{\prime} \rightarrow \eta \pi^{0} \pi^{0}$ is dominantly (and, as we have seen above, practically completely) due to $\pi \pi$ final-state rescattering. An even more interesting question, however, may be whether there is access to information on the $\pi \eta$ threshold parameters, too, in this decay. The observation in the last section that at least a large part of their effect can be absorbed in a redefinition of the tree-level couplings shows that this is not a trivial endeavor, and at least not in the center of the Dalitz plot.

The obvious question to ask is whether there is an interesting non-analytic behavior near the $\pi \eta$ thresholds, i.e. for $s_{1} / s_{2}$ close to $\left(M_{\eta}+M_{\pi}\right)^{2}$. In contrast to what makes the cusp at the $\pi^{+} \pi^{-}$threshold so special, we obviously cannot go below threshold and really see a cusp as a change in slope below vs. above a certain kinematical point; however, we still may look for square-root-like behavior at the border of the Dalitz plot, difficult as it would be to investigate such a phenomenon experimentally. From what we have learnt so far, such a cusp above threshold would have to be a two-loop effect and therefore is expected to be small. However, things turn our to be even worse: with the use of the threshold theorem again, applied now to the threshold $s_{1}=\left(M_{\eta}+M_{\pi}\right)^{2}$, one can show that the interference of genuine two-loop graphs with the tree-level amplitude (both real) is always exactly cancelled by the product of two corresponding one-loop graphs (both imaginary in our formalism), such that no square-root behavior survives in the squared amplitude [67]. This cancellation is shown 


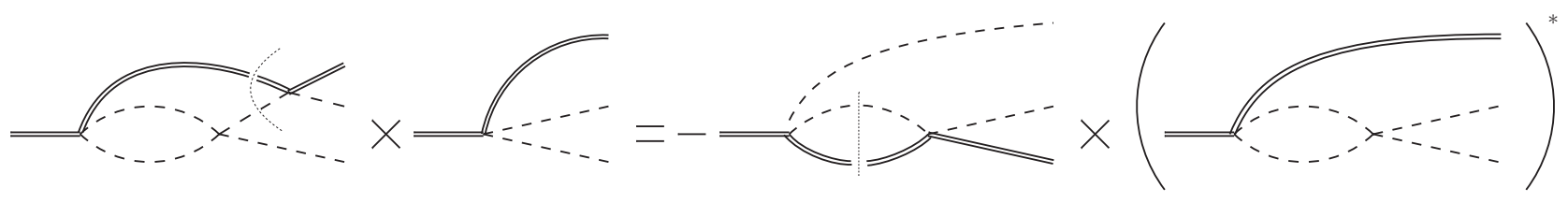

Fig. 14. Visualization of the cancellation of threshold cusps in $\eta^{\prime} \rightarrow \eta \pi^{0} \pi^{0}$ at $s_{1}=\left(M_{\eta}+M_{\pi^{0}}\right)^{2}$. Double lines denote both $\eta^{\prime}$ and $\eta$ fields, dashed lines stand for neutral pions. Figure taken from Ref. [67].

schematically in Fig. 14 for a specific set of graphs, and it can be shown to persist for all diagrams up to two loops. We therefore have to conclude that, with the methods described here, we cannot identify a method to extract $\pi \eta$ scattering lengths in a similar fashion as the cusp effect allows for the $\pi \pi$ ones.

\section{Summary and conclusions}

Non-relativistic effective field theory provides a systematic framework for an analysis of the cusp phenomenon and pion-pion scattering lengths in $K^{+} \rightarrow \pi^{0} \pi^{0} \pi^{+}$decays. The representation of the decay amplitude is calculated in a combined expansion in a non-relativistic parameter $\epsilon$ and $\pi \pi$ threshold parameters $a$, which is currently available up to $O\left(\epsilon^{4}, a \epsilon^{5}, a^{2} \epsilon^{4}\right)$. In order to match the enormous experimental accuracy achieved by the NA48/2 collaboration theoretically, radiative corrections have to be included. The effect of the latter on the $\pi \pi$ scattering lengths is surprisingly large, as photon effects modify the analytic structure of the decay amplitude near the $\pi^{+} \pi^{-}$threshold. Similar cusp phenomena are also present in other decays such as $K_{L} \rightarrow 3 \pi^{0}$ or $\eta \rightarrow 3 \pi^{0}$, where they are however far less prominent and much harder to use for a precision determination of scattering lengths. More promising in that respect is the decay $\eta^{\prime} \rightarrow \eta \pi^{0} \pi^{0}$, which, on the other hand, seems not to offer easy access to $\pi \eta$ threshold parameters.

\section{Acknowledgements}

I am very grateful to my coworkers Moritz Bissegger, Gilberto Colangelo, Andreas Fuhrer, Jürg Gasser, Akaki Rusetsky, and Sebastian Schneider for the most fruitful collaborations leading to the results presented here, and to Jürg Gasser for useful comments on this manuscript. I furthermore wish to thank Luigi Di Lella and Dmitri Madigozhin for providing me with the data for Figs. 1 and 9. We acknowledge the support of the European Community Research Infrastructure Integrating Activity "Study of Strongly Interacting Matter" (acronym HadronPhysics2, grant agreement No. 227431) under the Seventh Framework Programme of the EU. Work supported in part by DFG (SFB/TR 16, "Subnuclear Structure of Matter") and by the Helmholtz Association through funds provided to the virtual institute "Spin and strong QCD" (VH-VI-231).

\section{References}

1. S. Weinberg, Physica A 96 (1979) 327.

2. J. Gasser and H. Leutwyler, Annals Phys. 158 (1984) 142.

3. M. Gell-Mann, R. J. Oakes and B. Renner, Phys. Rev. 175 (1968) 2195.

4. M. Knecht, B. Moussallam, J. Stern and N. H. Fuchs, Nucl. Phys. B 457 (1995) 513 [arXiv:hep$\mathrm{ph} / 9507319]$.

5. J. F. Donoghue, J. Gasser and H. Leutwyler, Nucl. Phys. B 343 (1990) 341.

6. G. Colangelo, J. Gasser and H. Leutwyler, Nucl. Phys. B 603 (2001) 125 [arXiv:hep-ph/0103088].

7. G. Colangelo, J. Gasser and H. Leutwyler, Phys. Lett. B 488 (2000) 261 [arXiv:hep-ph/0007112].

8. B. Ananthanarayan, G. Colangelo, J. Gasser and H. Leutwyler, Phys. Rept. 353 (2001) 207 [arXiv:hep$\mathrm{ph} / 0005297]$.

9. S. Descotes-Genon, N. H. Fuchs, L. Girlanda and J. Stern, Eur. Phys. J. C 24 (2002) 469 [arXiv:hep$\mathrm{ph} / 0112088]$.

10. F. J. Ynduráin, R. García-Martín and J. R. Peláez, Phys. Rev. D 76 (2007) 074034 [arXiv:hepph/0701025].

11. R. Kamiński, J. R. Peláez and F. J. Ynduráin, Phys. Rev. D 77 (2008) 054015 [arXiv:0710.1150 [hep-ph]].

12. B. Adeva et al. [DIRAC Collaboration], Phys. Lett. B 619 (2005) 50 [arXiv:hep-ex/0504044].

13. S. Pislak et al., Phys. Rev. D 67 (2003) 072004 [arXiv:hep-ex/0301040].

14. J. R. Batley et al. [NA48/2 Collaboration], Eur. Phys. J. C 54 (2008) 411.

15. S. Deser, M. L. Goldberger, K. Baumann and W. E. Thirring, Phys. Rev. 96 (1954) 774.

16. A. Gall, J. Gasser, V. E. Lyubovitskij and A. Rusetsky, Phys. Lett. B 462 (1999) 335 [arXiv:hep-ph/9905309].

17. J. Gasser, V. E. Lyubovitskij, A. Rusetsky and A. Gall, Phys. Rev. D 64 (2001) 016008 [arXiv:hep$\mathrm{ph} / 0103157]$.

18. J. Gasser, V. E. Lyubovitskij and A. Rusetsky, Phys. Rept. 456 (2008) 167 [arXiv:0711.3522 [hep-ph]].

19. K. M. Watson, Phys. Rev. 88 (1952) 1163.

20. A. Pais and S. B. Treiman, Phys. Rev. 168 (1968) 1858.

21. G. Colangelo, J. Gasser and A. Rusetsky, Eur. Phys. J. C 59 (2009) 777 [arXiv:0811.0775 [hep-ph]].

22. J. R. Batley et al. [NA48/2 Collaboration], Phys. Lett. B 633 (2006) 173 [arXiv:hep-ex/0511056]. 
23. N. Cabibbo, Phys. Rev. Lett. 93 (2004) 121801 [arXiv:hep-ph/0405001].

24. E. P. Wigner, Phys. Rev. 73 (1948) 1002.

25. U.-G. Meißner, G. Müller and S. Steininger, Phys. Lett. B 406 (1997) 154 [Erratum-ibid. B 407 (1997) 454] [arXiv:hep-ph/9704377].

26. P. Budini and L. Fonda, Phys. Rev. Lett. 6 (1961) 419.

27. J. R. Batley et al. [NA48/2 Collaboration], Eur. Phys. J. C 64 (2009) 589 [arXiv:0912.2165 [hep-ex]].

28. N. Cabibbo and G. Isidori, JHEP 0503 (2005) 021 [arXiv:hep-ph/0502130].

29. E. Gámiz, J. Prades and I. Scimemi, Eur. Phys. J. C 50 (2007) 405 [arXiv:hep-ph/0602023].

30. G. Colangelo, J. Gasser, B. Kubis and A. Rusetsky, Phys. Lett. B 638 (2006) 187 [arXiv:hep-ph/0604084].

31. M. Beneke and V. A. Smirnov, Nucl. Phys. B 522 (1998) 321 [arXiv:hep-ph/9711391].

32. M. Knecht and R. Urech, Nucl. Phys. B 519 (1998) 329 [arXiv:hep-ph/9709348].

33. M. Bissegger, A. Fuhrer, J. Gasser, B. Kubis and A. Rusetsky, Phys. Lett. B 659 (2008) 576 [arXiv:0710.4456 [hep-ph]].

34. J. Gasser, B. Kubis and A. Rusetsky, in preparation.

35. L. D. Landau, Nucl. Phys. 13 (1959) 181.

36. R. Eden, P. V. Landshoff, D. I. Olive and J. C. Polkinghorne, The analytic S-matrix, Cambridge University Press (1966).

37. A. Nehme, Phys. Rev. D 70 (2004) 094025 [arXiv:hep-ph/0406209].

38. J. Bijnens and F. Borg, Eur. Phys. J. C 39 (2005) 347 [arXiv:hep-ph/0410333].

39. J. Bijnens and F. Borg, Eur. Phys. J. C 40 (2005) 383 [arXiv:hep-ph/0501163].

40. S. R. Gevorkyan, A. V. Tarasov and O. O. Voskresenskaya, Phys. Lett. B 649 (2007) 159 [arXiv:hepph/0612129].

41. S. R. Gevorkyan, D. T. Madigozhin, A. V. Tarasov and O. O. Voskresenskaya, Phys. Part. Nucl. Lett. 5 (2008) 85 [arXiv:hep-ph/0702154].

42. M. Bissegger, A. Fuhrer, J. Gasser, B. Kubis and A. Rusetsky, Nucl. Phys. B 806 (2009) 178 [arXiv:0807.0515 [hep-ph]].

43. G. Isidori, Eur. Phys. J. C 53 (2008) 567 [arXiv:0709.2439 [hep-ph]].

44. G. Gamow, Z. Phys. 51 (1928) 204.

45. A. Sommerfeld, Atombau und Spektrallinien, Volume 2, Vieweg, Braunschweig (1939).

46. J. Schwinger, J. Math. Phys. 5 (1964) 1606.
47. C. Ditsche, B. Kubis and U.-G. Meißner, Eur. Phys. J. C 60 (2009) 83 [arXiv:0812.0344 [hep-ph]].

48. C.-O. Gullström, A. Kupść and A. Rusetsky, Phys. Rev. C 79 (2009) 028201 [arXiv:0812.2371 [hep-ph]].

49. E. Abouzaid et al. [KTeV Collaboration], Phys. Rev. D 78 (2008) 032009 [arXiv:0806.3535 [hep-ex]].

50. C. Adolph et al. [WASA-at-COSY Collaboration], Phys. Lett. B 677 (2009) 24 [arXiv:0811.2763 [nuclex]].

51. M. Unverzagt et al. [Crystal-Ball-at-MAMI, TAPS, and A2 Collaborations], Eur. Phys. J. A 39 (2009) 169 [arXiv:0812.3324 [hep-ex]].

52. S. Prakhov et al. [Crystal-Ball-at-MAMI and A2 Collaborations], Phys. Rev. C 79 (2009) 035204 [arXiv:0812.1999 [hep-ex]].

53. D. Madigozhin, Pion scattering lengths from the cusp effect analysis, talk given at: FlaviAnet Kaon Workshop, Anacapri, Italy, June 12-14, 2008.

54. B. Kubis and S. P. Schneider, Eur. Phys. J. C 62 (2009) 511 [arXiv:0904.1320 [hep-ph]].

55. Project C.5 of the SFB/TR 16, "Subnuclear Structure of Matter", U. Wiedner, R. Beck et al.

56. A. Starostin, in Proc. 2nd Int. Workshop on Eta Meson Physics, Peñiscola, Spain, 2007, edited by M. Jacewicz and B. Höistad, arXiv:0710.1809 [nuclex].

57. P. Achenbach, arXiv:0802.2870 [nucl-ex].

58. M. Unverzagt [for the Crystal-Ball-at-MAMI Collaboration], arXiv:0910.1331 [hep-ex].

59. H. H. Adam et al. [WASA-at-COSY Collaboration], arXiv:nucl-ex/0411038.

60. B. R. Jany and D. Duniec [WASA-at-COSY Collaboration], Proc. 11th Int. Conf. on Meson-Nucleon Physics and the Structure of the Nucleon (MENU 2007), Jülich.

61. KLOE-2, Letter of Intent [http://www.lnf.infn.it/lnfadmin/direzione/roadmap/LoIKLOE.pdf].

62. C. Bloise, AIP Conf. Proc. 950 (2007) 192.

63. H. B. Li, J. Phys. G $36 \quad$ (2009) 085009 [arXiv:0902.3032 [hep-ex]].

64. V. Bernard, N. Kaiser and U.-G. Meißner, Phys. Rev. D 44 (1991) 3698.

65. M. Kolesár and J. Novotný, Eur. Phys. J. C 56 (2008) 231 [arXiv:0802.1289 [hep-ph]].

66. V. Dorofeev et al., Phys. Lett. B 651 (2007) 22 [arXiv:hep-ph/0607044].

67. S. P. Schneider and B. Kubis, PoS CD09 (2009) 120 [arXiv:0910.0200 [hep-ph]]. 